\title{
Clinical utility gene card for: Diamond Blackfan anemia
}

\author{
Adrianna Vlachos ${ }^{1}$, Niklas Dahl $^{2}$, Irma Dianzani ${ }^{3}$ and Jeffrey M Lipton ${ }^{\star, 1}$ \\ European Journal of Human Genetics (2011) 19; doi:10.1038/ejhg.2010.247; published online 19 January 2011
}

\section{DISEASE CHARACTERISTICS}

1.1 Name of the disease (synonyms)

Diamond Blackfan anemia, DBA1, 3-10 (DBA2 not confirmed), Aase Smith syndrome, congenital hypoplastic anemia, Blackfan Diamond anemia and inherited erythroblastopenia.

\subsection{OMIM\# of the disease}

$105650,610629,612527,612528,612561,612562,612563,613308$ and 613309.

1.3 Name of the analysed genes or DNA/chromosome segments RPS19, RPS24, RPS17, RPL35A, RPL5, RPL11, RPS7, RPS10 and RPS26.

1.4 OMIM\# of the gene(s)

$603474,602412,180472,180468,603634,604175,603658,603632$ and 603701.

\subsection{Mutational spectrum}

In patients for whom there is a known mutation (50-60\%), Diamond Blackfan anemia results from a ribosomal protein haploinsufficiency. ${ }^{1}$ It is likely that deletions, in addition to mutations, of the known genes associated with DBA account for an addition percentage of cases. ${ }^{2}$ No racial or ethnic predilection has been identified. The relative frequency of affected ribosomal genes identified is $\sim 25 \%, R P S 19 ; 2 \%, R P S 24$; 1\%, RPS17; 1-2\%, RPL35A; 7\%, RPL5; 5-10\%, RPL11; 2\%, RPS7; $<1-2 \%, R P S 10$; and $6 \%, R P S 26 .^{2-7}$ Mutations resulting in haploinsufficiency or loss of function in all nine genes thus far described include missense mutations, nonsense mutations, splice mutations, insertions, deletions and rearrangements. ${ }^{8,9}$

\subsection{Analytical methods}

Depending on the nature and magnitude of the sequence change a variety of techniques are available: cytogenetics for large deletions and rearrangements, multiple ligation dependent analysis (MLPA) for detection of large deletions and rearrangements, array comparative genomic hybridization (CGH) for detection of deletions, quantitative PCR and genomic sequencing. It is estimated that $\sim 10 \%$ of RPS 19 haploinsufficient DBA cases are caused by large deletions that are missed by exon sequencing. ${ }^{10}$

\subsection{Analytical validation}

The analysis from genetic tests should be performed by experienced and well-educated personnel in certified diagnostic laboratories.
Technical errors, sample mix-up and natural genetic variations may in rare cases create misinterpretations. To avoid this, analysis and validation should be performed by professionals in environments with established routines.

\subsection{Estimated frequency of the disease}

(incidence at birth ('birth prevalence') or population prevalence)

The incidence is from 5 to 10 per one million live births. ${ }^{11}$

1.9 If applicable, prevalence in the ethnic group of investigated person

There is no known ethnic predilection or exclusion.

1.10 Diagnostic setting

\begin{tabular}{lcc}
\hline & Yes & No \\
\hline A. (Differential) diagnostics & $\bigotimes$ & $\square$ \\
B. Predictive testing & $\bigotimes$ & $\square$ \\
C. Risk assessment in relatives & $\bigotimes$ & $\square$ \\
D. Prenatal & $\bigotimes$ & $\square$ \\
\hline
\end{tabular}

\section{Comment:}

In addition, genetic testing has been used in the following diagnostic settings.

E. Related stem cell transplant donor screening to be certain that the donor is not affected.

\section{TEST CHARACTERISTICS}

\begin{tabular}{|c|c|c|c|c|}
\hline & \multicolumn{2}{|c|}{ Genotype or disease } & \multirow{2}{*}{$\begin{array}{l}\text { A: True positives } \\
\text { B: False positives }\end{array}$} & \multirow{2}{*}{$\begin{array}{l}\text { C: False negative } \\
\text { D: True negative }\end{array}$} \\
\hline & Present & Absent & & \\
\hline \multicolumn{5}{|l|}{ Test } \\
\hline Positive & A & $\mathrm{B}$ & $\begin{array}{l}\text { Sensitivity: } \\
\text { Specificity: }\end{array}$ & $\begin{array}{l}A /(A+C) \\
D /(D+B)\end{array}$ \\
\hline Negative & C & $\mathrm{D}$ & $\begin{array}{l}\text { Positive predictive value: } \\
\text { Negative predictive value: }\end{array}$ & $\begin{array}{l}A /(A+B) \\
D /(C+D)\end{array}$ \\
\hline
\end{tabular}

\subsection{Analytical sensitivity}

(proportion of positive tests if the genotype is present)

Nearly $100 \%$, within the margin of laboratory error (this applies to genetic testing only)

\footnotetext{
${ }^{1}$ Feinstein Institute for Medical Research, Hofstra North Shore-LIJ School of Medicine, Division of Hematology/Oncology and Stem Cell Transplantation, Steven and Alexandra Cohen Children's Medical Center of New York, New Hyde Park, NY, USA; ${ }^{2}$ Department of Genetics and Pathology, Uppsala University, Uppsala, Sweden; ${ }^{3}$ Department of Medical Sciences, Eastern Piedmont University, Novara, Italy

${ }^{*}$ Correspondence: Dr JM Lipton, Feinstein Institute for Medical Research, Hofstra North Shore-LIJ School of Medicine, Steven and Alexandra Cohen Children's Medical Center of New York, 269-01 76th Street, New Hyde Park, NY 11040, USA. Tel: +1 718470 3470; Fax: +1 718 343 4642; E-mail: jlipton@nshs.edu
} 
2.2 Analytical specificity

(proportion of negative tests if the genotype is not present)

Nearly $100 \%$, within the margin of laboratory error (this applies to genetic testing only)

\subsection{Clinical sensitivity}

(proportion of positive tests if the disease is present)

Currently approximately $50-60 \%$ of clinically diagnosed cases can be identified by mutation analysis, MLPA, ${ }^{12}$ or array-based screening for copy number variations (eg, CGH).

\subsection{Clinical specificity}

(proportion of negative tests if the disease is not present)

Close to $100 \%$. False positives because of the technical or clerical errors are possible, if the disease, in the broadest sense is not present, the test will generally be negative. The penetrance and expressivity of DBA is quite variable, thus, there are instances, even in multiplex families, in which a genetically affected individual has subtle or no evidence of clinical disease. Therefore, the definition of what is disease is critical. ${ }^{12}$ In this instance a clinically unaffected individual could have a positive test. Clinicians, patients/families and geneticists may define 'presence of disease' quite differently.

\subsection{Positive clinical predictive value}

(life-time risk to develop the disease if the test is positive)

Again the definition of what constitutes disease (see 2.4) is critical. There are not enough data to fully predict the 'natural history' in non-expressing (clinically unaffected) individuals with known DBAcausing mutations or deletions that result in ribosomal protein haploinsufficiency. The majority of such individuals do have some evidence of disease, although these manifestations can be subtle. A minority of patients with known genotypes have no discernible clinical or hematological evidence of DBA.

\subsection{Negative clinical predictive value} (probability not to develop the disease if the test is negative) Index case in that family had been tested:

If the proband is positive for a mutation, virtually $100 \%$ Index case in that family had not been tested:

A non-affected first degree relative to a (non-tested) index case has an indeterminate risk, as only $50-60 \%$ of the patients have a known DBA-associated ribosomal gene mutation or deletion.

\section{CLINICAL UTILITY}

3.1 (Differential) diagnosis: The tested person is clinically affected 3.1.1 Can a diagnosis be made other than through a genetic test?

\begin{tabular}{|c|c|c|}
\hline No & (continue with 3.1 .4 ) & $\square$ \\
\hline \multirow[t]{7}{*}{ Yes } & $\otimes$ & \\
\hline & Clinically & $\otimes$ \\
\hline & Imaging & $\square$ \\
\hline & Endoscopy & $\square$ \\
\hline & Biochemistry & $\nabla^{*}$ \\
\hline & Electrophysiology & $\square$ \\
\hline & Other (please describe) & $\square$ \\
\hline
\end{tabular}

*Elevated fetal hemoglobin and/or erythrocyte adenosine deaminase activity, whereas not diagnostic for DBA, can be supportive of the diagnosis in the appropriate clinical setting. ${ }^{13} \mathrm{~A}$ number of research methods, most importantly Northern blot analysis to determine the presence of characteristic ribosomal RNA processing defects, may ultimately be available as a diagnostic screening tool. However, these tests are currently not available for clinical use.

\subsubsection{Describe the burden of alternative diagnostic methods to the patient.}

A clinical diagnosis is based on a history, physical examination, laboratory tests that require venipuncture, and a bone marrow aspirate, which may be performed under anesthesia or deep conscious sedation. There is a minimal but incremental risk for anesthesia and the aspirate, which include pain, bleeding and rarely $(\sim 1: 50000)$ death. Occasionally ultrasound or radiological imaging is required to determine the presence of certain congenital anomalies supportive of the diagnosis of DBA.

\subsubsection{How is the cost effectiveness of alternative diagnostic methods to be judged?}

There are no studies available to determine the cost effectiveness of any diagnostic approach to DBA. Costs will differ depending on the laboratory fees and imaging required. The consequence of missing the diagnosis or failing to determine the presence or absence of particular hematological or non-hematological manifestations of DBA may be significant.

3.1.4 Will disease management be influenced by the result of a genetic test?

No

Yes

Therapy: stem cell transplantation requires a non-DBA-affected donor. ${ }^{14,15}$ This can only be confirmed by determining the absence of a DBA-associated gene abnormality in the prospective related donor.

Prognosis: there are no genotype-phenotype correlations that are predictive of prognosis, that is, response to corticosteroid therapy, likelihood of remission, cancer predisposition (occurrence or type of cancer) or life expectancy.

Management: management is dependent on the response to treatment with corticosteroids. Patients who respond to tolerable doses can be maintained on corticosteroids. Those patients that do not respond or cannot be maintained on tolerable doses are placed on a chronic red cell transfusion/iron chelation protocol. Management of iron balance is critical for transfused patients. Stem cell transplantation is reserved for patients with transfusion dependence and a matched related or unrelated donor, and for those patients who develop clinically significant neutropenia, thrombocytopenia, leukemia or myelodysplastic syndrome. At this time there is no genotype-phenotype correlation for the development of other bone marrow complications. Identification and correction of birth defects is also essential. There are some reported genotype-phenotype correlations with regard to orofacial anomalies (ie, cleft palate) and skeletal anomalies. ${ }^{6,16}$

3.2 Predictive setting: The tested person is clinically unaffected but carries an increased risk based on family history

Yes.

Mildly affected or unaffected carriers should be informed about subclinical expression of the disease (eg, subnormal $\mathrm{Hb}$ levels), recurrence risks in next generation and complications associated with pregnancies. A regular check of $\mathrm{Hb}$ levels may be recommended. 


\subsubsection{Will the result of a genetic test influence lifestyle and prevention?}

If the test result is positive (please describe):

Yes.

In a family, either multiplex or identified through an affected proband, a positive test in a family member will impart the burden of the disease. This individual may be variably affected, and in those cases in which a clinical diagnosis is obvious, a positive test will just confirm the diagnosis. When there is no, or only minimal, evidence of the disease, a positive genetic test will confirm that the individual may transmit the mutation to offspring. Reproductive options can be pursued that may decrease the likelihood of having an affected child. ${ }^{17,18}$ The positive individual would also be disqualified as a stem cell transplant donor, regardless of histocompatibility, even if the individual was clinically unaffected. ${ }^{14}$

If the test result is negative (please describe):

Yes.

In a family, either multiplex or identified through an affected proband, a negative test will relieve the burden of the disease from the negative individual, remove the necessity for any reproductive options related to the transmission of the autosomal dominant disorder, and allow the individual to be a stem cell transplant donor based upon histocompatibility.

\subsubsection{Which options in view of lifestyle and prevention does a person at-risk have if no genetic test has been done?}

For clinically affected individuals, genetic testing provides the advantage of confirmation of diagnosis, as other marrow failure syndromes may share significant clinical overlap. The identification of a specific gene involved has no advantage to the individual as no relevant genotype-phenotype correlations have been established. However, in the absence of testing, reproductive options cannot be established for the prevention of transmission of the disease to the next generation.

\subsection{Genetic risk assessment in family members of a diseased person}

Risk assessment is preferably performed on an individual basis and related to the family structure, clinical investigations and a combination of biochemical and genetic test results.

\subsubsection{Does the result of a genetic test resolve the genetic situation in that family?}

Yes, if a DBA-associated ribosomal protein gene mutation or deletion has been found in the family (a mutation or deletion has only been identified in $\sim 60 \%$ of families).

\subsubsection{Can a genetic test in the index patient save genetic or other tests in family members?}

Yes, if it is positive for a known DBA-associated ribosomal protein gene mutation or deletion, only that gene needs to be tested.

\subsubsection{Does a positive genetic test result in the index patient enable a predictive test in a family member? \\ Yes.}

\subsection{Prenatal diagnosis}

Yes. Prenatal testing may be offered if a genetic marker is identified in an affected family member. This may in some cases also be combined with HLA typing in order to have a matched sibling for stem cell transplantation.
3.4.1 Does a positive genetic test result in the index patient enable a prenatal diagnostic?

Yes, in almost all instances. There is a small possibility of gonadal mosaicism in which a child with a positive test is born to parents with a negative test. ${ }^{19}$

\section{IF APPLICABLE, FURTHER CONSEQUENCES OF TESTING}

Please assume that the result of a genetic test has no immediate medical consequences. Is there any evidence that a genetic test is nevertheless useful for the patient or his/her relatives?

Yes, genetic testing of the parents or other relatives of an affected child will allow reproductive choices that may reduce or eliminate the possibility of vertical transmission of DBA. As well, positive genetic testing helps remove any doubt about the diagnosis.

\section{CONFLICT OF INTEREST}

The authors declare no conflict of interest.

\section{ACKNOWLEDGEMENTS}

This work was supported by the EuroGentest, an EU-FP6 supported NoE, contract number 512148 (EuroGentest Unit 3: 'Clinical genetics, community genetics and public health', Workpackage 3.2) and grants from the Daniella Maria Arturi Foundation (JML, AV), Pediatric Cancer Foundation (JML), National Institutes of Health R01 HL 079571-06 (JML, AV), The Feinstein Institute for Medical Research General Clinical Research Center M01 RR018535 (JML, AV), Telethon (ID), DBA Foundation (ID), Italian Ministry of University and Research (ID), Gruppo di Sostegno DBA Italia (ID) and the Swedish Research Council (ND).

1 Gazda HT, Zhong R, Long L et al: RNA and protein evidence for haplo-insufficiency in Diamond-Blackfan anaemia patients with RPS19 mutations. Br J Haematol 2004; 127: 105-113.

2 Draptchinskaia N, Gustavsson P, Andersson B et al: The gene encoding ribosomal protein S19 is mutated in Diamond-Blackfan anaemia. Nat Genet 1999; 21: 169-175.

3 Gazda HT, Grabowska A, Merida-Long LB et al: Ribosomal protein S24 gene is mutated in Diamond-Blackfan anemia. Am J Hum Genet 2006; 79: 1110-1118.

4 Cmejla R, Cmejlova J, Handrkova H, Petrak J, Pospisilova D: Ribosomal protein S17 gene (RPS17) is mutated in Diamond-Blackfan anemia. Hum Mutat 2007; 28 : 1178-1182.

5 Farrar JE, Nater M, Caywood $\mathrm{E}$ et al: Abnormalities of the large ribosomal subunit protein, Rpl35a, in Diamond-Blackfan anemia. Blood 2008; 112: 1582-1592.

6 Gazda HT, Sheen MR, Vlachos A et al: Ribosomal protein L5 and L11 mutations are associated with cleft palate and abnormal thumbs in Diamond-Blackfan anemia patients. Am J Hum Genet 2008; 83: 769-780.

7 Doherty L, Sheen MR, Vlachos A et al: Ribosomal protein genes RPS10 and RPS26 Are commonly mutated in Diamond-Blackfan anemia. Am J Hum Genet 2010; 86: 222-228.

8 Boria I, Quarello P, Avondo F et al: A new database for ribosomal protein genes which are mutated in Diamond-Blackfan Anemia. Hum Mutat 2008; 29: E263-E270.

9 Campagnoli MF, Ramenghi U, Armiraglio M et al: RPS19 mutations in patients with Diamond-Blackfan anemia. Hum Mutat 2008; 29: 911-920. Review.

10 Boria I, Garelli E, Gazda HT et al: The ribosomal basis of Diamond-Blackfan anemia: mutation and database update. Hum Mutat 2010; 31: 1269-1279.

11 Willig TN, Niemeyer CM, Leblanc T et al: Identification of new prognosis factors from the clinical and epidemiologic analysis of a registry of 229 Diamond-Blackfan anemia patients. DBA group of Société d'Hématologie et d'Immunologie Pédiatrique (SHIP), Gesellshaft für Pädiatrische Onkologie und Hämatologie (GPOH) and the European Society for Pediatric Hematology and Immunology (ESPHI). Pediatr Res 1999; 46: 553-561.

12 Quarello P, Garelli E, Brusco A et al: Multiplex ligation-dependent probe amplification enhances molecular diagnosis of Diamond-Blackfan anemia due to RPS19 deficiency. Haematologica 2008; 93: 1748-1750.

13 Vlachos A, Ball S, Dahl N, et al: Participants of Sixth Annual Daniella Maria Arturi International Consensus Conference: Diagnosing and treating Diamond Blackfan anaemia: results of an international clinical consensus conference. $\mathrm{Br} J$ Haematol 2008; 142: 859-876.

14 Orfali RF, Wynn RF, Stevens RF, Chopra R, Ball SE: Failure of red cell production following allogenic BMT for Diamond Blackfan anaemia (DBA) illustrates functional significance of high erythrocyte adenosine deaminase (eADA) activity in the donor. Blood 1999; 94: 414a. 
15 Vlachos A, Federman N, Reyes-Haley C, Abramson J, Lipton JM: Hematopoietic stem cell transplantation for Diamond Blackfan anemia: a report from the Diamond Blackfan Anemia Registry. Bone Marrow Transplant 2001; 27: 381-386.

16 Quarello P, Garelli E, Carando A et al: Diamond-Blackfan anemia: genotype-phenotype correlations in Italian patients with RPL5 and RPL11 mutations. Haematologica 2010; 95: 206-213.

17 Samuel GN, Strong KA, Kerridge I, Jordens CF, Ankeny RA, Shaw PJ: Establishing the role of pre-implantation genetic diagnosis with human leucocyte antigen typing: what place do 'saviour siblings' have in paediatric transplantation? Arch Dis Child 2009; 94 : 317-320.

18 Kuliev A, Rechitsky S, Tur-Kaspa I, Verlinsky Y: Preimplantation genetics: improving access to stem cell therapy. Ann NY Acad Sci 2005; 1054: 223-227.

19 Cmejla R, Blafkova J, Stopka T et al: Ribosomal protein S19 gene mutations in patients with diamond-blackfan anemia and identification of ribosomal protein S19 pseudogenes. Blood Cells Mol Dis 2000; 26: 124-132. 